\title{
Symmetry for the duration of entropy-consuming intervals
}

\author{
Reinaldo García-García* and Daniel Domínguez \\ Centro Atómico Bariloche and Instituto Balseiro, 8400 San Carlos de Bariloche, Río Negro, Argentina \\ (Received 26 September 2013; revised manuscript received 11 March 2014; published 14 May 2014)
}

\begin{abstract}
We introduce the violation fraction $v$ as the cumulative fraction of time that a mesoscopic system spends consuming entropy at a single trajectory in phase space. We show that the fluctuations of this quantity are described in terms of a symmetry relation reminiscent of fluctuation theorems, which involve a function $\Phi$, which can be interpreted as an entropy associated with the fluctuations of the violation fraction. The function $\Phi$, when evaluated for arbitrary stochastic realizations of the violation fraction, is odd upon the symmetry transformations that are relevant for the associated stochastic entropy production. This fact leads to a detailed fluctuation theorem for the probability density function of $\Phi$. We study the steady-state limit of this symmetry in the paradigmatic case of a colloidal particle dragged by optical tweezers through an aqueous solution. Finally, we briefly discuss possible applications of our results for the estimation of free-energy differences from single-molecule experiments.
\end{abstract}

DOI: 10.1103/PhysRevE.89.052121

PACS number(s): 05.70.Ln, 05.40.-a

\section{INTRODUCTION}

Stochastic thermodynamics is currently a very active field given its great relevance for physics, chemistry, and biology [1]. As a consequence of fluctuations, which are extremely important in mesoscopic systems, the energy change, work, heat, and entropy production associated with any process exhibit a stochastic nature, i.e., their values are random quantities that depend on the particular trajectory the system follows in phase space. The fluctuations of some thermodynamic observables are constrained to satisfy general relations, which are known as fluctuation theorems [2-8], that arise as a consequence of two main properties: ergodicity and microreversibility. These relations can be generally written as

$$
\ln \frac{P(S)}{P^{\mathcal{T}}(-S)}=S,
$$

where $P(S)$ is the probability density function of the stochastic observable $S$ representing a given form of trajectory-dependent entropy production, while $\mathcal{T}$ represents a transformation, usually time reversal, the transformation to a dual dynamics, or the composition of these two operations (see [9-11] for a simple definition of the dual dynamics). The quantity $S$ exhibits the symmetry $S^{\mathcal{T}}=-S$.

In the past few years, the applicability of these relations has also been extended to systems exhibiting stochastic nonMarkovian dynamics [12-18], as well as being widely tested in experiments [19-24]. Fluctuation theorems are consistent: The second law of thermodynamics, the Green-Kubo formula, and the Onsager reciprocity relations, for example, can be deduced from them.

The stochastic nature of thermodynamic observables may lead to a negative value of the entropy production for particular realizations of a given process. In fact, the occurrence of such rare realizations is exponentially less probable than the occurrence of trajectories compatible with the second law of thermodynamics (i.e., realizations where the entropy production is positive), as can be immediately interpreted from Eq. (1). It is important to remark, however, that the stochastic

\footnotetext{
*reinaldomeister@gmail.com
}

entropy production is not restricted to being positive, i.e., the occurrence of negative values of $S$ at a single trajectory in phase space does not represent a violation of the second law of thermodynamics, which states that the average $\langle S\rangle$ is non-negative.

In other matters, if we focus on a single trajectory of the system in phase space, one finds that for certain time intervals the entropy production is negative. The statistics of the cumulative duration of these time intervals has been recently studied [25]. There, this duration (relative to the total duration of the process) was denominated the violation fraction and the occurrence of negative values of the entropy production was referred to as local violations of the second law of thermodynamics, following the terminology of Ref. [19] (the term local meaning at a single trajectory and stating that not true violations of the second law occur). The study made in Ref. [25] is, however, incomplete. First, instead of the full probability density function of the violation fraction, only its first moment was studied. Second, some particular conditions were assumed, e.g., the system was assumed to be prepared in a steady state and to be connected to a single reservoir.

The aim of the present paper is to fill these gaps. To this aim, we derive in this work a general symmetry relation for the probability density function of the violation fraction. This relation is valid under very general conditions: The system may be prepared in an arbitrary initial state and connected to one or several thermal baths. No special assumptions are needed for the results derived in this paper to hold, only ergodicity and microreversibility. ${ }^{1}$ Our results are rather general and also hold for arbitrary external protocols.

This symmetry relation is reminiscent of fluctuation theorems and involves a function $\Phi$ that reflects the asymmetry between forward and transformed processes in phase space as regards the violation fraction. This function satisfies, by construction, respective integral and detailed fluctuation theorems and is also odd upon some relevant transformations,

\footnotetext{
${ }^{1}$ This statement is valid if we consider Markovian dynamics. When considering non-Markovian dynamics, ergodicity and microreversibility would not be enough. At least stability should be additionally demanded, as discussed in Ref. [18].
} 
similar to the entropy production. Thus, it may in principle be interpreted as an entropy. On the other hand, the average of $\Phi$ represents a lower bound for the average of the entropy production, i.e., the inequality $\langle S\rangle \geqslant\langle\Phi\rangle \geqslant 0$ holds quite generally.

At this point we would like to clarify a subtle but important issue. In Ref. [25] the violation fraction (denoted therein by $v$ ) was defined in terms of the entropy produced from the beginning of the process $S$, i.e., the cumulative fraction of time; the entropy production from the beginning of the process was negative. This definition is not invariant upon time translations, i.e., measuring the entropy production with respect to different time instants leads to different sets of violation intervals. Here we consider the stochastic entropy production rate $\sigma=d S / d t$ instead of the stochastic entropy production in order to define the local violations of the second law. Then, within the present framework, a violation sector is defined as a time interval where $\sigma<0$.

The rest of the paper is organized as follows. In the next section we derive a symmetry relation for the probability density function of the violation fraction in both the transient and stationary regimes. We also discuss the main properties and deepen the physical interpretation of the function $\Phi$ involved in the referred symmetry. In Sec. III we study the steady-state limit of the referred symmetry for a paradigmatic model system. We determine the large-deviation function associated with the violation fraction in that limit, providing both particular analytical and general numerical results. In Sec. IV we discuss the relevance of our study and possible applications in the estimation of free energies from singlemolecule experiments. We provide a summary and concluding remarks in Sec. V.

\section{SYMMETRY FOR THE PROBABILITY DENSITY FUNCTION OF THE VIOLATION FRACTION}

\section{A. Transient symmetry}

We start by anticipating the main result of the present section. If we introduce the probability density function for the violation fraction $v(\tau)$ [see Eq. (9)] to be in the vicinity of the value $v$ at time $\tau$ for the forward $\rho(v, \tau)$ and transformed $\rho^{\mathcal{T}}(v, \tau)$ processes (see details below), we obtain the symmetry relation

$$
\ln \frac{\rho(v, \tau)}{\rho^{\mathcal{T}}(1-v, \tau)}=\Phi(v, \tau) .
$$

The function $\Phi$ is odd upon the corresponding transformation $\mathcal{T}$. Moreover, making a parallel with classical thermodynamics, $\Phi$ can be seen as an entropy associated with the local violations of the second law, a sort of superentropy, as we discuss below. Already at this point we note from Eq. (2) that the integral fluctuation theorem

$$
\left\langle e^{-\Phi(v, \tau)}\right\rangle=\int_{0}^{1} d v \rho(v, \tau) e^{-\Phi(v, \tau)}=1
$$

holds at all times $\tau$. From this and Jensen's inequality $-\ln x \geqslant$ $1-x$ for $x>0$, we see that a law similar to the second law of thermodynamics holds for $\Phi,\langle\Phi(v, \tau)\rangle \geqslant 0$, a result that can also be seen by identifying the average of $\Phi$ with the positively defined Kullback-Leibler distance between the distributions $\rho$ and $\rho^{\mathcal{T}},\langle\Phi(v, \tau)\rangle=\mathcal{D}_{\mathrm{KL}}\left(\rho \| \rho^{\mathcal{T}}\right)$, where

$$
\mathcal{D}_{\mathrm{KL}}\left(\rho \| \rho^{\mathcal{T}}\right)=\int_{0}^{1} d v \rho(v, \tau) \ln \frac{\rho(v, \tau)}{\rho^{\mathcal{T}}(1-v, \tau)} .
$$

This quasi-second law of thermodynamics for $\Phi$ imposes strong restrictions on the stochastic process $v(\tau)$. We remark that some minimal requirements are needed for the entropy production associated with a given process to satisfy the second law of thermodynamics, ergodicity being one of them. It is thus intriguing that the fraction of time a process spends consuming entropy also exhibits those requirements.

We focus on systems relaxing to well-defined steady states for constant parameters. It is worth noting the distinction between two different types of systems: those where detailed balance holds and the steady-state probability density function corresponds to the Boltzmann-Gibbs distribution and those relaxing to a nonequilibrium steady state (NESS) driven by nonconservative forces and/or special boundary conditions. It is known [26] that for the latter family of systems the total entropy production $S_{\text {tot }}$ splits into an adiabatic contribution $S_{\text {a }}$ accounting for the energy dissipated in maintaining a NESS and a nonadiabatic contribution $S_{\text {na }}$ that accounts for the relaxation to the steady state and for the effect of an external driving. Each of these three forms of entropy production satisfies a fluctuation theorem in terms of the corresponding symmetry operation [time reversal (R) for $S_{\text {tot }}$, dual transformation $(\dagger)$ for $S_{\mathrm{a}}$, and their composition $(\dagger \circ \mathrm{R})$ for $S_{\text {na }}$ ], leading to three different faces of the second law of thermodynamics [27]. Given that our results are valid for each of these forms of entropy production, we generically write $v, S$, and $\sigma$ without further specification, understanding the notation $\mathcal{T}$ as the corresponding symmetry transformation associated with each case.

Let us consider an ergodic and microreversible system driven by a set of external parameters, which we denote by $\lambda_{t}$. The state of the system in phase space is denoted by the symbol $m$, which may be a discrete state or a continuous variable, vector, or field. A trajectory in phase space from $t=0$ to the final time $t=\tau$ is denoted by a bold symbol $\mathbf{m}=\{m(t)\}_{t=0}^{\tau}$, while the full time dependence of the protocol is denoted by $\lambda_{\tau}=\left\{\lambda_{t}\right\}_{t=0}^{\tau}$. Additionally, we introduce the time-reversed trajectory $\mathbf{m}^{\mathrm{R}}=\{m(\tau-t)\}_{t=0}^{\tau}$ and protocol $\lambda_{\tau}^{\mathrm{R}}=\left\{\lambda_{\tau-t}\right\}_{t=0}^{\tau}$. As the evolution is stochastic, one can define the probability weights for trajectories $\mathcal{P}_{\tau}\left[\mathbf{m} ; \lambda_{\tau}\right]$. A key aspect of ergodic and microreversible systems is that the trajectory-dependent entropy production may be written as the log-ratio of path probability weights

$$
\begin{gathered}
S_{\mathrm{tot}}\left[\mathbf{m}, \lambda_{\tau}\right]=\ln \frac{\mathcal{P}_{\tau}\left[\mathbf{m} ; \lambda_{\tau}\right]}{\mathcal{P}_{\tau}^{\mathrm{R}}\left[\mathbf{m}^{\mathrm{R}} ; \lambda_{\tau}^{\mathrm{R}}\right]}, \\
S_{\mathrm{na}}\left[\mathbf{m}, \lambda_{\tau}\right]=\ln \frac{\mathcal{P}_{\tau}\left[\mathbf{m} ; \lambda_{\tau}\right]}{\mathcal{P}_{\tau}^{\dagger \mathrm{R}}\left[\mathbf{m}^{\mathrm{R}} ; \lambda_{\tau}^{\mathrm{R}}\right]}, \\
S_{\mathrm{a}}\left[\mathbf{m}, \lambda_{\tau}\right]=\ln \frac{\mathcal{P}_{\tau}\left[\mathbf{m} ; \lambda_{\tau}\right]}{\mathcal{P}_{\tau}^{\dagger}\left[\mathbf{m} ; \lambda_{\tau}\right]},
\end{gathered}
$$

leading to the fluctuation theorems for arbitrary protocols, initial conditions, and number of reservoirs [26]. On the other hand, given an arbitrary observable $\mathcal{O}\left[\mathbf{m} ; \lambda_{\tau}\right]$, the following 
equivalences hold [11]:

$$
\begin{aligned}
\left\langle\mathcal{O}\left[\mathbf{m} ; \lambda_{\tau}\right]\right\rangle & =\left\langle\left.\mathcal{O}\left[\mathbf{m}^{\mathrm{R}} ; \lambda_{\tau}\right] e^{-S_{\text {tot }}^{\mathrm{R}}\left[\mathbf{m}, \lambda_{\tau}^{\mathrm{R}} g\right]}\right|^{\mathrm{R}}\right. \\
& =\left\langle\mathcal{O}\left[\mathbf{m}^{\mathrm{R}} ; \lambda_{\tau}\right] e^{-S_{\mathrm{na}}^{\dagger \mathrm{oR}}\left[\mathbf{m}, \lambda_{\tau}^{\mathrm{R}}\right]}\right\rangle^{\dagger \circ \mathrm{R}} \\
& =\left\langle\mathcal{O}\left[\mathbf{m} ; \lambda_{\tau}\right] e^{-S_{\mathrm{a}}^{\dagger}\left[\mathbf{m}, \lambda_{\tau}\right]}\right\rangle^{\dagger},
\end{aligned}
$$

where $\langle\cdots\rangle^{\mathcal{T}}$ denotes the average with the transformed probability weight $\mathcal{P}_{\tau}^{\mathcal{T}}$, for $\mathcal{T}=\mathrm{R}, \dagger \circ \mathrm{R}$, and $\dagger$, respectively. Let us now formally introduce the violation fraction for a given trajectory

$$
v(\tau) \stackrel{\text { def }}{=} \frac{1}{\tau} \int_{0}^{\tau} \Theta(-\sigma(t)) d t
$$

where $\Theta(\cdots)$ is the Heaviside step function and we have suppressed, for simplicity of notation, the full dependence on the particular trajectory in phase space and the protocol. A key point for our derivations is that the violation fraction satisfies the symmetry

$$
v^{\mathcal{T}}(\tau)=1-v(\tau)
$$

which can be seen as a direct consequence of microreversibility and ergodicity, similar to the symmetries that the different forms of entropy production exhibit upon the corresponding operations. $^{2}$

Let us now introduce the joint probability density function for the violation fraction to exhibit a value in the interval $[v, v+d v]$ having observed a value of the entropy production in the interval $[S, S+d S]$ at time $\tau, P(v, S, \tau)$, and the corresponding transformed probability density function $P^{\mathcal{T}}(v, S, \tau)$ given, respectively, by the expressions

$$
\begin{gathered}
P(v, S, \tau)=\langle\delta(v-v(\tau)) \delta(S-S(\tau))\rangle, \\
P^{\mathcal{T}}(v, S, \tau)=\left\langle\delta\left(v-v^{\mathcal{T}}(\tau)\right) \delta\left(S-S^{\mathcal{T}}(\tau)\right)\right\rangle^{\mathcal{T}} .
\end{gathered}
$$

Using the previous definitions (8) and (10) and recalling that $S$ is odd upon the operation $\mathcal{T}$, we can write

$$
P(v, S, \tau)=P^{\mathcal{T}}(1-v,-S, \tau) e^{S} .
$$

Our main result (2) follows by identifying the probability density function for the violation fraction to be in the vicinity of the value $v$ at time $\tau$, as $\rho(v, \tau)=\int d S P(v, S, \tau)$, while the transformed probability density function is given by

$$
\rho^{\mathcal{T}}(1-v, \tau)=\int d S P^{\mathcal{T}}(1-v, S, \tau)=\int d S P(v, S, \tau) e^{-S},
$$

where we have used a change of variables $S \rightarrow-S$ and the symmetry (13). Introducing now the conditional probability

\footnotetext{
${ }^{2}$ For the adiabatic entropy production rate, Eq. (10) holds since $S(\tau)=-S^{\dagger}(\tau)$ and $S(\tau)=\int_{0}^{\tau} d t \sigma(t)$, implying that $\sigma(t)=-\sigma^{\dagger}(t)$. In the remaining cases, where a time-reversal operation is involved, Eq. (10) follows from recalling that the entropy production rate is, for Markovian dynamics, a local function of coordinates and protocols $\sigma(t)=\sigma(m(t), \dot{m}(t) ; \lambda(t))$, which changes sign when evaluated for time-reversed arguments.
}

density function for the entropy production to be in the vicinity of $S$ at time $\tau$ given that the observed value of the violation fraction was $v, P(S, \tau \mid v)=P(v, S, \tau) / \rho(v, \tau)$, we obtain Eq. (2), with the identification

$$
\Phi(v, \tau)=-\ln \left\langle e^{-S(\tau)} \mid v\right\rangle=-\ln \int d S P(S, \tau \mid v) e^{-S} .
$$

Before closing this section we would like to mention some important issues. First, we note that the existing fluctuation theorems are exact relations for the probability density function of stochastic observables $\mathcal{O}$ exhibiting the symmetry relation $\mathcal{O}^{\mathcal{T}}=-\mathcal{O}$. The observable considered here, the violation fraction, exhibits, in contrast, a symmetry of the form $\mathcal{O}^{\mathcal{T}}=1-\mathcal{O}$. If instead of considering the violation fraction (9) we study the related magnetization [28] $\psi(\tau)$ given by

$$
\psi(\tau) \stackrel{\text { def }}{=} \frac{1}{\tau} \int_{0}^{\tau} \operatorname{sgn}(\sigma(t)) d t
$$

where $\operatorname{sgn}(\cdots)$ is the sign function, we recover an observable that is odd upon the generic transformation $\mathcal{T}$. In this case, we can easily obtain a symmetry relation for $\psi$ using our main result (2) and the trivial identity $\psi=1-2 v$.

We note, however, that the referred symmetry could also be derived from first principles by following the same lines of reasoning leading to (2). It is important to note that the violation fraction and the magnetization are time-averaged quantities, thus the symmetry for the probability density function of $\psi$ is a stochastic version of the functional Crooks theorem derived in Ref. [29] for time averages of arbitrary phase-space functions in the case of deterministic dynamics starting at equilibrium. In our case the observable is stochastic, as is the evolution of the system in phase space, and we consider arbitrary initial conditions and symmetry transformations.

\section{B. Steady-state symmetry}

When the system asymptotically relaxes to a NESS such that the mean value of the entropy production rate tends to a constant value, one can derive a steady-state symmetry for the probability density function of the violation fraction in the same way that a steady-state fluctuation theorem holds for the entropy production. This is relevant, for example, when considering small Brownian motors operating under steadystate conditions or systems relaxing to a NESS after a fast quench. Another interesting case that falls into this category is that of a system driven at a constant rate, for which the nonadiabatic entropy production rate approaches a constant value at large times. The necessary condition is that the limit

$$
\lim _{\tau \rightarrow \infty} P(\sigma, \tau)=P_{\infty}(\sigma)
$$

exists unambiguously, where, as usual, $\sigma$ could be any particular form of entropy production rate.

Let us introduce the quantity $R(\tau)=\operatorname{Prob}[\sigma(\tau)<0]$. From Eq. (9) we have

$$
\langle v(\tau)\rangle=\frac{1}{\tau} \int_{0}^{\tau} R(t) d t
$$


Assuming that there is a finite characteristic relaxation time $\tau_{r}$ to reach the NESS, one can see that one has

$$
\begin{aligned}
\left\langle v\left(\tau \gg \tau_{r}\right)\right\rangle & =\frac{1}{\tau} \int_{0}^{\tau_{r}} R(t) d t+\frac{1}{\tau} \int_{\tau_{r}}^{\tau} R(t) d t \\
& \approx \frac{1}{\tau} \int_{0}^{\tau_{r}} R(t) d t+R_{\infty}\left(1-\frac{\tau_{r}}{\tau}\right) \\
& =v_{\infty}+\frac{A_{\infty}}{\tau},
\end{aligned}
$$

to the first nonvanishing order, where $v_{\infty}=R_{\infty}, A_{\infty}=$ $\int_{0}^{\tau_{r}} R(t) d t-R_{\infty} \tau_{r}$, and

$$
R_{\infty}=\lim _{\tau \rightarrow \infty} R(\tau)=\int_{-\infty}^{0} P_{\infty}(\sigma) d \sigma .
$$

We note here the finite value of $\langle v(\infty)\rangle=v_{\infty}$, in contrast to the case studied in Ref. [25] in terms of the entropy production $S$ instead of the entropy production rate. In that case, it was shown that the average violation fraction vanishes for $\tau \rightarrow \infty$. However, it is worth noting that to leading order both quantities relax as $\tau^{-1}$ for large $\tau$, except, for instance, in the vicinity of a critical point where the large fluctuations may lead to a different asymptotic behavior [25]. Additionally, we remark that for $\tau \rightarrow \infty$ the violation fraction converges in density to its mean, since the system is ergodic:

$$
\begin{aligned}
\lim _{\tau \rightarrow \infty} v(\tau) & =\lim _{\tau \rightarrow \infty} \frac{1}{\tau} \int_{0}^{\tau} \Theta(-\sigma(t)) d t \\
& =\langle\Theta(-\sigma(t))\rangle_{\mathrm{ss}} \equiv v_{\infty},
\end{aligned}
$$

where $\langle\cdots\rangle_{\mathrm{ss}}$ represents the average in the steady state.

We note that if the system is asymptotically stationary, one expects that the long-time behavior of the probability density function of the violation fraction may be well described in terms of a large deviation function $\zeta(v)$ :

$$
\rho(v, \tau) \sim e^{-\zeta(v) \tau}
$$

The same behavior is expected for $\Phi$, which means that we can write $\Phi(v, \tau) \rightarrow \phi(v) \tau$ for $\tau \rightarrow \infty$, with

$$
\phi(v)=-\lim _{\tau \rightarrow \infty} \frac{1}{\tau} \ln \left\langle e^{-S(\tau)} \mid v\right\rangle .
$$

From the previous reasoning, given that in the stationary limit the operation $\mathcal{T}$ is meaningless, we obtain the steady-state symmetry

$$
\zeta(1-v)-\zeta(v)=\phi(v)
$$

\section{Physical properties of the function $\Phi$}

Let us study in more detail the main physical properties of the function $\Phi$ for arbitrary systems submitted to arbitrary protocols. The interpretation of this function is by no means exclusive. This means that, if we consider any other functional of trajectories in phase space instead of the violation fraction, the corresponding asymmetry function will share the same general properties of $\Phi$. However, given that the violation fraction measures how likely the consumption of entropy is, this characterization is relevant.

We start by noting that, from the definition given by Eq. (15), the function $\Phi$ is related to the average of $e^{-S}$ restricted to those trajectories with a fixed value of the violation fraction $v$. Then we can establish a link with classical thermodynamics that clarifies the physical meaning of this function. Note that, identifying $v$ as an energy and $S$ as a coordinate, the conditional probability $P(S, \tau \mid v)$ can be seen as a microcanonical distribution (where energy is fixed) and the conditional average $\left\langle e^{-S} \mid v\right\rangle$ as a sort of inverse phase-space volume at fixed energy. Then $\Phi(v, \tau)$ is the microcanonical entropy linked to the energy $v$. In this case, the equal $a$ priori probability postulate needs the weight $e^{-S}$, which serves as a balance, since those microstates with negative values of $S$ (i.e., those trajectories producing negative entropy) are exponentially less probable.

We now continue by proving that the average of $\Phi$ represents a lower bound for the entropy production. First, note that the definition given by Eq. (15) can be rewritten as

$$
\left\langle e^{-(S-\Phi(v, \tau))} \mid v\right\rangle=1
$$

From the previous expression, the following conditional inequality holds:

$$
\langle S(\tau) \mid v\rangle \geqslant \Phi(v, \tau)
$$

Multiplying both terms of Eq. (26) by $\rho(v, \tau)$ and integrating $v$ out, we obtain

$$
\langle S(\tau)\rangle \geqslant\langle\Phi(v, \tau)\rangle
$$

It is straightforward to see that $\Phi$ is odd upon the corresponding operation $\mathcal{T}$. Indeed, introducing the transformed potential $\Phi^{\mathcal{T}}(v, \tau)=-\ln \left\langle e^{-S} \mid v\right\rangle^{\mathcal{T}}$, we have

$$
\begin{aligned}
\Phi^{\mathcal{T}}(1-v, \tau) & =-\ln \int d S P^{\mathcal{T}}(S, \tau \mid 1-v) e^{-S} \\
& =-\ln \int d S \frac{P^{\mathcal{T}}(1-v, S, \tau)}{\rho^{\mathcal{T}}(1-v, \tau)} e^{-S} \\
& =-\ln \int d S \frac{P^{\mathcal{T}}(1-v,-S, \tau)}{\rho(v, \tau)} e^{S} e^{\Phi(v, \tau)} \\
& =-\Phi(v, \tau)-\ln \int d S P(S, \tau \mid v) \\
& \equiv-\Phi(v, \tau)
\end{aligned}
$$

where we have used Eqs. (2) and (13), a change of variables $S \rightarrow-S$, and the definition of the conditional probability and its normalization condition $\int d S P(S, \tau \mid v)=1$. Let us introduce the probability density function for the values of $\Phi$, $P(\Phi, \tau)$, and its transformed counterpart $P^{\mathcal{T}}(\Phi, \tau)$ as follows:

$$
\begin{gathered}
P(\Phi, \tau)=\int_{0}^{1} d v \delta(\Phi-\Phi(v, \tau)) \rho(v, \tau), \\
P^{\mathcal{T}}(\Phi, \tau)=\int_{0}^{1} d v \delta\left(\Phi-\Phi^{\mathcal{T}}(v, \tau)\right) \rho^{\mathcal{T}}(v, \tau) .
\end{gathered}
$$

Then, using the previous definitions complemented by Eqs. (2) and (28), we obtain that a detailed fluctuation theorem also holds for $\Phi$ :

$$
\ln \frac{P(\Phi, \tau)}{P^{\mathcal{T}}(-\Phi, \tau)}=\Phi
$$


All these properties strongly support the interpretation of $\Phi$ as an entropy associated with the fluctuations of the violation fraction.

Having defined the function $\Phi$ by Eq. (15), we now derive an alternative formula to determine this quantity that provides more insight into its physical meaning. If we divide both terms of Eq. (13) by $\rho(v, \tau)$ and use Eq. (2), we obtain

$$
P(S, \tau \mid v)=P^{\mathcal{T}}(-S, \tau \mid 1-v) e^{S-\Phi(v, \tau)},
$$

from which we immediately get

$$
\Phi(v, \tau)=S-\ln \frac{P(S, \tau \mid v)}{P^{\mathcal{T}}(-S, \tau \mid 1-v)} .
$$

This expression is valid for any value of $S$, but it turns into a very meaningful formula when we consider the case of $S=0$ :

$$
\Phi(v, \tau)=-\ln \frac{P(0, \tau \mid v)}{P^{\mathcal{T}}(0, \tau \mid 1-v)} .
$$

This alternative definition with a focus on trajectories that do not produce entropy provides a clear interpretation of $\Phi$ and allows us to show that $\Phi(1 / 2, \tau) \equiv 0$ irrespective of the particular protocols and of the value of $\tau$, as we discuss below.

Let us denote the full space of possible trajectories in phase space by $\Omega$ and let us introduce the set $\mathbb{V}_{\lambda}=\{\mathbf{m} \in \Omega \mid v(\tau)=$ $1 / 2$ \} for a given protocol $\lambda_{\tau}$, which can be arbitrarily chosen. Let us also introduce the twin set $\mathbb{V}_{\lambda^{\tau}}$, corresponding to the transformed protocol $\lambda_{\tau}^{\mathcal{T}}$ and the transformed dynamics. It is worth noting that, by virtue of Eq. (10), the set $\mathbb{V}_{\lambda}$ maps onto $\mathbb{V}_{\lambda} \mathcal{T}$ under the transformation $\mathcal{T}$. Indeed, given that $v=1 / 2$ if and only if $v=v^{\mathcal{T}}$, we see that for any trajectory $\mathbf{m} \in \mathbb{V}_{\lambda}$, we have that $\mathbf{m}^{\mathcal{T}} \in \mathbb{V}_{\lambda^{\mathcal{T}}}$ also. On the other hand, if $\mathbf{m} \notin \mathbb{V}_{\lambda}$, then $\mathbf{m}^{\mathcal{T}} \notin \mathbb{V}_{\lambda^{T}}$ either.

This, however, does not hold for arbitrary subsets of $\mathbb{V}_{\lambda}$. In particular, let us introduce the parametrized family of subsets $\mathbb{S}_{\lambda}(S)$ of $\mathbb{V}_{\lambda}$ as $\mathbb{S}_{\lambda}(S)=\left\{\mathbf{m} \in \mathbb{V}_{\lambda} \mid S(\tau)=S\right\}$ and the corresponding family under the transformed dynamics $\mathbb{S}_{\lambda^{T}}(S)=\left\{\mathbf{m} \in \mathbb{V}_{\lambda^{\tau}} \mid S(\tau)=S\right\}$. Then, given that the entropy production satisfies the symmetry $S^{\mathcal{T}}=-S$, we have that for any $S, \mathbb{S}_{\lambda}(S)$ maps onto $\mathbb{S}_{\lambda^{T}}(-S)$ under $\mathcal{T}$ :

$$
\mathbb{S}_{\lambda}(S) \stackrel{\mathcal{T}}{\longmapsto} \mathbb{S}_{\lambda^{\mathcal{T}}}(-S) .
$$

A special case is one for which $S=0$, because from Eq. (35) we see that $\mathbb{S}_{\lambda}(0) \stackrel{\mathcal{T}}{\longmapsto} \mathbb{S}_{\lambda} \tau(0)$. In particular, if $\mathbf{m} \in \mathbb{S}_{\lambda}(0)$ and its probability weight is $\mathcal{P}[\mathbf{m} ; \lambda]$, it is easy to see that $\mathbf{m}^{\mathcal{T}} \in$ $\mathbb{S}_{\lambda^{\mathcal{T}}}(0)$ also and that the probability weight of $\mathbf{m}^{\mathcal{T}}$ is also $\mathcal{P}[\mathbf{m} ; \lambda]$ (i.e., $\mathcal{P}[\mathbf{m} ; \lambda]=\mathcal{P}^{\mathcal{T}}\left[\mathbf{m}^{\mathcal{T}} ; \lambda^{\mathcal{T}}\right]$ ) because $S=0$. Then we may write

$$
P(0, \tau \mid 1 / 2)=P^{\mathcal{T}}(0, \tau \mid 1 / 2) .
$$

Evaluating Eq. (34) for $v=1 / 2$ and using Eq. (36), we immediately obtain $\Phi(1 / 2, \tau)=0$.

We finish this section by noting that, by virtue of the second law of thermodynamics, the small values of the violation fraction are more likely than the large values of this quantity. For small values of $v, \Phi$ is positive, while for large values of $v, \Phi$ is negative. We conjecture that $\Phi(v, \tau)$ must quite generally be a decreasing function of $v$ for $v \in[0,1]$, which means that $v=1 / 2$ is the only zero of $\Phi$ and that $\Phi(v, \tau)$ admits an inverse function.

\section{LARGE-DEVIATION FUNCTION FOR THE VIOLATION FRACTION: A CASE STUDY}

\section{A. Posing of the problem}

In this section we study and determine the large-deviation function of the violation fraction in the paradigmatic case of an overdamped colloidal particle dragged through a viscous fluid by an optical tweezers with a harmonic potential

$$
V(x ; \lambda)=\frac{1}{2}(x-\lambda)^{2},
$$

where the focus of the optical tweezers is moved at a constant rate $b, \lambda(t)=b t$. Although being widely studied, this example is still instructive. On the other hand, even in this simple case the derivation of a closed analytical solution is not possible. The system evolves under the Langevin dynamics

$$
\dot{x}(t)=-[x(t)-b t]+\sqrt{2 T} \xi(t),
$$

where the white noise $\xi(t)$ has zero mean and variance $\left\langle\xi(t) \xi\left(t^{\prime}\right)\right\rangle=\delta\left(t-t^{\prime}\right)$. If the system is initially prepared in the steady state associated with $\lambda(0)=0$, the stochastic entropy production corresponds in this case to the Jarzynski work [6]: ${ }^{3}$

$$
S(t)=\int_{0}^{t} \dot{\lambda}\left(t^{\prime}\right) \partial_{\lambda} V\left(x\left(t^{\prime}\right) ; \lambda\left(t^{\prime}\right)\right) d t^{\prime},
$$

from where the stochastic entropy production rate can be identified as

$$
\sigma(t)=\frac{b}{T}[b t-x(t)]
$$

Let us introduce a new stochastic process $\eta(t)$ as

$$
\eta(t)=\frac{1}{\sqrt{2 T}}[b t-x(t)]-\eta_{m},
$$

with $\eta_{m}=b / \sqrt{2 T}$. Then the equation of motion for this process reads

$$
\dot{\eta}(t)=-\eta(t)+\xi(t) .
$$

Note that one has that $\sigma(t)<0$ if and only if $\eta+\eta_{m}<0$, which means that the violation fraction for our problem can be written in terms of the auxiliary process $\eta(t)$ as

$$
v(\tau)=\frac{1}{\tau} \int_{0}^{\tau} \Theta\left(-\eta(t)-\eta_{m}\right) d t .
$$

The statistics of the occupation times associated with the Ornstein-Uhlenbeck process given by Eq. (42) have been widely studied in the literature (see, for instance, Ref. [30]). Furthermore, there is a well-established method to compute the large-deviation function associated with any nonlinear functional of $\eta[31,32]$. We briefly review the method below, as presented in Ref. [31].

\footnotetext{
${ }^{3}$ An additional term involving the change in free energy $\Delta F(t)$ is generally present, however, in this case the free energy does not depend on $\lambda$ and one only needs to consider the thermodynamic work.
} 


\section{B. Large-deviation function for arbitrary time-averaged quantities}

The probability distribution of the process $\eta(t)$ for $0 \leqslant t \leqslant$ $\tau$ is given by

$$
\mathcal{P}[\eta]=\mathcal{N} \exp \left\{-\frac{1}{2} \int_{0}^{\tau}[\dot{\eta}(t)+\eta(t)]^{2} d t\right\},
$$

where $\mathcal{N}$ is a normalization constant. We are interested in the probability density function of the time-averaged quantity

$$
r(\tau)=\frac{1}{\tau} \int_{0}^{\tau} U_{0}(\eta(t)) d t
$$

where $U_{0}(\eta)$ is an arbitrary function of the stochastic variable $\eta$. In practice it is convenient to look at the distribution $P_{u}(u)$ of the quantity $u=r \tau$. Its Laplace transform reads

$$
\hat{P}_{u}(s)=\langle\exp (-r s \tau)\rangle=Z(s) / Z(0),
$$

with

$$
Z(s)=\int \mathcal{D}[\eta] \exp \left\{-\frac{1}{2} \int_{0}^{\tau}\left[\dot{\eta}^{2}+2 \eta \dot{\eta}+\eta^{2}+2 s U_{0}(\eta)\right] d t\right\} .
$$

We are interested in the limit $\tau \rightarrow \infty$. It is convenient to impose periodic boundary conditions $\eta(\tau)=\eta(0)$ since this restriction will not change the results in the large- $\tau$ limit. With this we can drop the term $2 \eta \dot{\eta}$, which is a perfect derivative. Then $Z(s)$ is the imaginary-time Feynman path integral that gives the partition function of a quantum particle with Hamiltonian $H=p^{2} / 2+\eta^{2} / 2+s U_{0}(\eta)$ at inverse temperature $\tau$, $p$ being the canonical momentum conjugate to $X$. For $\tau \rightarrow \infty$ the ground state dominates:

$$
\langle\exp (-r s \tau)\rangle=\exp \left\{-\tau\left[E_{g}(s)-E_{g}(0)\right]\right\},
$$

where $E_{g}(s)$ is the ground-state energy for the Schrödinger equation

$$
-\frac{1}{2} \frac{d^{2} \psi(\eta)}{d \eta^{2}}+U(\eta, s) \psi(\eta)=E(s) \psi(\eta),
$$

with

$$
U(\eta, s)=\frac{\eta^{2}}{2}+s U_{0}(\eta) .
$$

For $s=0$ the problem reduces to a simple harmonic oscillator and $E_{g}(0)=1 / 2$. We now note that, from Eq. (48), the large-time behavior of $P(r, \tau)$ is given by the inverse Laplace transform

$$
P(r, \tau) \propto \int_{-i \infty}^{i \infty} d s \exp [\tau g(s)]
$$

where $g(s)=r s+E_{g}(0)-E_{g}(s)$. Using the steepest-descent method, one sees that we have $P(r, \tau) \approx \exp [-\zeta(r) \tau]$, with

$$
\zeta(r)=\max _{s}\left[E_{g}(s)-E_{g}(0)-r s\right] .
$$

We now use this method to compute the large-deviation function of the violation fraction associated with the process given by Eq. (38).

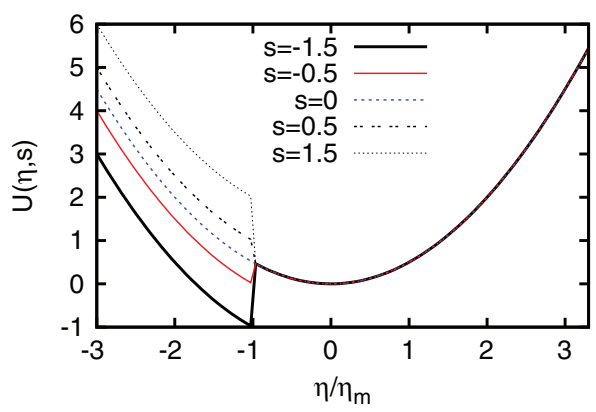

FIG. 1. (Color online) Effective potential for equivalent quantum problem associated with the large-deviation function of the violation fraction, for different values of $s$. At $\eta=-\eta_{m}$, the parabolic potential exhibits a jump of magnitude $U\left(-\eta_{m}^{+}, s\right)-U\left(-\eta_{m}^{-}, s\right)=-s$.

\section{Large-deviation function for the violation fraction}

From Eq. (43) we see that for the violation fraction the effective potential reads

$$
U(\eta, s)=\frac{\eta^{2}}{2}+s \Theta\left(-\eta-\eta_{m}\right) .
$$

In Fig. 1 we plot this potential for different values of $s$ to explicitly show its shape. At $\eta=-\eta_{m}$, the parabolic potential exhibits a jump of magnitude $-s$.

We note that in our problem the Schrödinger equation acquires the following particular form:

$$
\begin{gathered}
\psi_{+}^{\prime \prime}-\eta^{2} \psi_{+}+2 E(s) \psi_{+}=0, \\
\psi_{-}^{\prime \prime}-\eta^{2} \psi_{-}-2 s \psi_{-}+2 E(s) \psi_{-}=0,
\end{gathered}
$$

where $\psi_{+}(\eta)=\psi\left(\eta>-\eta_{m}\right), \quad \psi_{-}(\eta)=\psi\left(\eta<-\eta_{m}\right)$, and $\psi^{\prime \prime}=d^{2} \psi / d \eta^{2}$. As in Ref. [31], the solutions of Eqs. (54) and (55) can be expressed in terms of parabolic cylinder functions $D_{p}(z)$ using the standard solutions of the parabolic cylinder equation $y^{\prime \prime}-\left(z^{2} / 4+a\right) y=0$. Selecting the solutions that satisfy the physical boundary condition $\psi( \pm \infty)=0$ gives

$$
\begin{gathered}
\psi_{+}(\eta)=A D_{p^{+}}(\sqrt{2} \eta), \\
\psi_{-}(\eta)=B D_{p^{-}}(-\sqrt{2} \eta),
\end{gathered}
$$

where $A$ and $B$ are normalization constants, while $p^{+}$and $p^{-}$ are given by

$$
\begin{gathered}
p^{+}=E(s)-\frac{1}{2}, \\
p^{-}=E(s)-\frac{1}{2}-s .
\end{gathered}
$$

Imposing the continuity of $\psi$ and $\psi^{\prime}$ at $\eta=-\eta_{m}$, we obtain the following eigenvalue equation for $E(s)$ :

$$
\frac{D_{p^{+}}^{\prime}\left(-\sqrt{2} \eta_{m}\right)}{D_{p^{+}}\left(-\sqrt{2} \eta_{m}\right)}=-\frac{D_{p^{-}}^{\prime}\left(\sqrt{2} \eta_{m}\right)}{D_{p^{-}}\left(\sqrt{2} \eta_{m}\right)}
$$

from which the ground-state energy $E_{g}(s)$ and all the excited states can be obtained. In general, this problem cannot be solved analytically for generic values of $s$ and $\eta_{m}$, so a 
numerical solution of the eigenvalue problem (60) is mandatory. Nevertheless, certain interesting limits can be studied analytically, as we discuss below.

\section{The limit $v \rightarrow 0$}

In the limit $v \rightarrow 0$ the violation sector $\eta<-\eta_{m}$ is almost inaccessible, which means, appealing to the reader's physical intuition, that this scenario is compatible with an infinite wall at $\eta=-\eta_{m}$. The present limit corresponds then to the case $s \rightarrow+\infty$.

It has recently been shown that in the case of an infinite wall displaced to the left of the center of a parabolic potential, the ground-state energy $\epsilon_{0}\left(\eta_{m}\right)$ decreases monotonically as a function of the center-wall distance [33]. Thus, for a hard wall at $\eta=-\eta_{m}$, we have $E_{g}(+\infty)=\epsilon_{0}\left(\eta_{m}\right)$, with $1 / 2<\epsilon_{0}\left(\eta_{m}\right) \leqslant 3 / 2$ and $d \epsilon_{0}\left(\eta_{m}\right) / d \eta_{m}<0$. The ground-state energy $\epsilon_{0}\left(\eta_{m}\right)$ has to be determined numerically for arbitrary $\eta_{m}[33,34]$.

We need the first correction to this result for large $s$. Although it can be derived formally, we will continue by using physical arguments to render calculations easier and to enlighten the discussion.

First, note that for $s \rightarrow \infty$ the details of the quadratic potential are not important in Eq. (55) and we can see the problem as an equivalent problem with a high potential barrier (of magnitude $s$ ) for $\eta<-\eta_{m}$. In this case the ground-state wave function penetrates the barrier by a typical depth $\delta_{g}^{+}(s)=$ $\left\{2\left[s-E_{g}(s)\right]\right\}^{-1 / 2} \approx(2 s)^{-1 / 2}$, where it drops to zero. The problem is then equivalent, in physical terms, to a problem with an infinite hard wall but placed at $-\eta_{\delta}=-\eta_{m}-\delta_{g}^{+}(s)$. Note that in the equivalent model the wave function identically vanishes at $\eta=-\eta_{\delta}$. We can then write, to leading order in $\delta_{g}^{+}(s)$

$$
E_{g}(s)=\epsilon_{0}\left(\eta_{m}\right)-\left|\epsilon_{0}^{\prime}\left(\eta_{m}\right)\right|(2 s)^{-1 / 2} .
$$

This equation coincides [without dropping the term $E_{g}(s) \approx$ $E_{g}(\infty)$ from the square root] with the formal result obtained in Ref. [34] by means of perturbation theory. The formal equivalence can be easily seen by using the virial theorem given by Eq. (15) of Ref. [33]. Using now Eq. (61) and the general method given by Eq. (52), we obtain in the limit $v \rightarrow 0$

$$
\zeta(v)=\zeta_{0}-a_{0} v^{1 / 3},
$$

with $\zeta_{0}=\epsilon_{0}\left(\eta_{m}\right)-1 / 2$ and $a_{0}=\frac{3}{2}\left|\epsilon_{0}^{\prime}\left(\eta_{m}\right)\right|^{2 / 3}$. In the limit of slow driving $\eta_{m} \ll 1$, we can write a closed expression for $\zeta(v)$ to first order in $\eta_{m}$. Using $\epsilon_{0}(0)=3 / 2$ and $\left|\epsilon_{0}^{\prime}(0)\right|=$ $2 / \sqrt{\pi}$ [34], we have

$$
\zeta(v)=-\frac{2}{\sqrt{\pi}} \eta_{m}+\zeta_{\mathrm{qs}}(v),
$$

where the quasistatic large-deviation function $\zeta_{\mathrm{qs}}(v)$ is given by

$$
\zeta_{\mathrm{qs}}(v)=1-\frac{3}{2}\left(\frac{4}{\pi} v\right)^{1 / 3},
$$

which is the expected result for $\eta_{m}=0$. The last statement can be seen by considering the magnetization $\psi$. Substituting in Eq. (64) the identity $v=(1-\psi) / 2$ and noting that for $v \rightarrow 0$ we have that $\psi \rightarrow 1$, we obtain exactly Eq. (60) of Ref. [31].

\section{The limit $v \rightarrow 1$}

We now study the limit of large values of the violation fraction. In this limit, trajectories spend most of the time in the violation sector $\eta<-\eta_{m}$. Then the right branch of the parabolic potential is almost inaccessible, a situation that is compatible with the limit $s \rightarrow-\infty$. In this case, the potential has a deep minimum at $\eta=-\eta_{m}$, with energy $\eta_{m}^{2} / 2+s$, thus it is convenient to redefine $E(s)=s+\varepsilon(s)$. With this, Eqs. (54) and (55) read

$$
\begin{gathered}
\psi_{+}^{\prime \prime}-\eta^{2} \psi_{+}+2 s \psi+2 \varepsilon(s) \psi_{+}=0, \\
\psi_{-}^{\prime \prime}-\eta^{2} \psi_{-}+2 \varepsilon(s) \psi_{-}=0 .
\end{gathered}
$$

We now note that, for $s \rightarrow-\infty$, the details of the quadratic potential are not important in Eq. (65). Then, just as we did in the limit $v \rightarrow 0$, we can neglect the effect of the parabolic potential in (65) and consider a high potential barrier of height $-s$. With this, the ground-state wave function penetrates the region $\eta>-\eta_{m}$ by a small depth $\delta_{g}^{-}(s) \approx(-2 s)^{-1 / 2}$ and we can approximate our problem with an equivalent one with a hard wall at $\eta=-\eta_{m}+\delta_{g}^{-}(s)$. For $\eta<-\eta_{m}$, the solution of Eq. (66) is still given by a parabolic cylinder function

$$
\psi_{-}(\eta)=A D_{p}(-\sqrt{2} \eta)
$$

where $A$ is a normalization constant and $p=\varepsilon(s)-1 / 2$. The hard-wall condition gives the eigenvalue equation for $\varepsilon(s)$ :

$$
D_{p}\left(\sqrt{2}\left[\eta_{m}-\delta_{g}^{-}(s)\right]\right)=0
$$

Equation (68) still has to be solved numerically, however, the limit of slow driving $\eta_{m} \ll 1$ can be treated analytically. For any small $\eta$, we can write $D_{p}(-\sqrt{2} \eta) \approx D_{p}(0)-\sqrt{2} D_{p}^{\prime}(0) \eta$. With this, we can rewrite our eigenvalue equation as

$$
\frac{D_{p}(0)}{D_{p}^{\prime}(0)}=\sqrt{2} \eta_{m}-(-s)^{-1 / 2} .
$$

Given that the right-hand side of Eq. (69) is a small quantity, we can expand $\varepsilon(s)=3 / 2-\epsilon$ for the ground state, with $\epsilon \ll$ 1. Using standard identities relating the parabolic cylinder functions to $\Gamma$ functions [35], we have

$$
\frac{\Gamma\left(-\frac{p}{2}\right)}{\Gamma\left(\frac{1-p}{2}\right)}=\sqrt{2}(-s)^{-1 / 2}-2 \eta_{m} .
$$

Note that $p=1-\epsilon$. Expanding the $\Gamma$ functions above for small $\epsilon$, we have $\Gamma((\epsilon-1) / 2) / \Gamma(\epsilon / 2) \approx-\sqrt{\pi} \epsilon$, thus we obtain

$$
\epsilon=\frac{2}{\sqrt{\pi}} \eta_{m}-\sqrt{-\frac{2}{\pi s}} .
$$

We then have for the ground-state energy

$$
E_{g}(s)=s+\frac{3}{2}-\frac{2}{\sqrt{\pi}} \eta_{m}+\sqrt{-\frac{2}{\pi s}},
$$




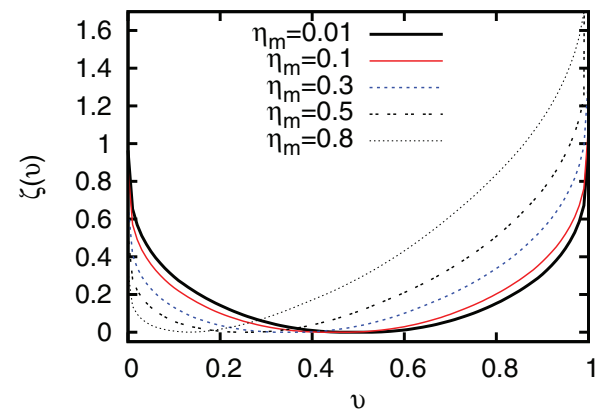

FIG. 2. (Color online) Large-deviation function for the violation fraction for $\eta_{m}=0.01,0.1,0.3,0.5$, and 0.8 . For $\eta_{m}=0.01$ the large-deviation function is very symmetric with respect to $v=1 / 2$. The position of the minimum of this function decreases very rapidly as $\eta_{m}$ increases.

from which we obtain for the large-deviation function, in the $\operatorname{limit} v \rightarrow 1$,

$$
\zeta(v)=-\frac{2}{\sqrt{\pi}} \eta_{m}+\zeta_{\mathrm{qs}}(1-v),
$$

with $\zeta_{\text {qs }}$ again given by Eq. (64). We point out that, again, for $\eta_{m}=0$ and using $1-v=(1+\psi) / 2$ in terms of the magnetization $\psi$, we obtain Eq. (59) of Ref. [31].

We would like to remark that the behavior of $\zeta(v)$ is, from Eqs. (63) and (73), similar for $v \rightarrow 0$ and $v \rightarrow 1$. On the other hand, one expects these behaviors to be different for nonzero $\eta_{m}$ since the second law of thermodynamics favors small values of the violation fraction and penalizes large values of this quantity. Our analytical results show that the difference in the behavior around $v \rightarrow 0$ with respect to the behavior around $v \rightarrow 1$ is at least of second order of perturbation theory in $\eta_{m}$, around $\eta_{m}=0$. Thus, for small, still finite values of $\eta_{m}$, one expects $\zeta(v)$ to be very symmetric around $v=1 / 2$, exactly as for $\eta_{m}=0$.

\section{General results}

We now turn to the numerical solution of Eq. (60). In Fig. 2 we plot the large-deviation function obtained numerically. It can be seen that for $\eta_{m}=0.01$ the large-deviation function is very symmetric, a fact that is in concordance with our analytical results (63) and (73). As $\eta_{m}$ increases, the position of the minimum of the large-deviation function decreases very rapidly while this function becomes very asymmetric, even for $\eta_{m}<1$. This fact can be understood as follows. From the dynamics given by Eq. (38) and the definition of the entropy production rate (40) we obtain that in the stationary limit the probability density function of $\sigma$ reads

$$
P_{\infty}(\sigma)=\frac{1}{2 \sqrt{\pi} \eta_{m}} \exp \left[-\frac{1}{4 \eta_{m}^{2}}\left(\sigma-2 \eta_{m}^{2}\right)^{2}\right] .
$$

From this result, we get that the mean value of the violation fraction in this limit is given by

$$
\langle v(\tau)\rangle=\int_{-\infty}^{0} P_{\infty}(\sigma) d \sigma=\frac{1}{2} \operatorname{erfc}\left(\eta_{m}\right),
$$

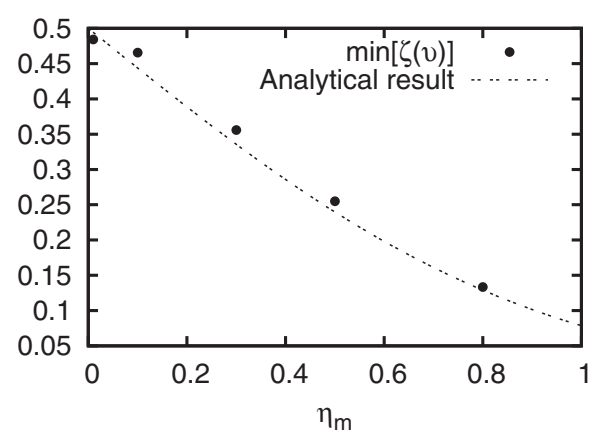

FIG. 3. Comparison between the numerically obtained position of the minimum of $\zeta(v)$, for different values of $\eta_{m}$, and the exact result given by Eq. (75). Both results coincide within the numerical errors.

where $\operatorname{erfc}(\cdot \cdots)$ is the complementary error function. Recalling that the position of the minimum of the large-deviation function corresponds to $\langle v\rangle$, which explains why this point shifts so rapidly to the left when we increase $\eta_{m}$. In Fig. 3 we plot the numerically obtained position of the minimum of the large-deviation function and the exact result given by Eq. (75), obtaining very good agreement between both results within the numerical errors.

The asymmetry function $\phi(v)$ can be obtained directly from Eq. (24). We plot this function in Fig. 4. For $\eta_{m}=0.01$ this function is almost flat, since in this case the thermal fluctuations are large and/or the driving velocity is small (recall the definition of $\eta_{m}$ ), which means that the local violations of the second law are more probable in this limit. As the driving velocity increases (and/or the temperature decreases), the asymmetry between small and large values of the violation fraction increases very rapidly.

This is easy to understand. Note that, from the exact result given by Eq. (74), we see that $\langle\sigma\rangle=2 \eta_{m}^{2}$, while $\sqrt{2\left\langle\delta \sigma^{2}\right\rangle}=2 \eta_{m}$, with $\delta \sigma(t)=\sigma(t)-\langle\sigma(t)\rangle$. Then, as long as the amplitude of the fluctuations is greater than $\langle\sigma\rangle$, the local violations are likely to occur, but if $\sqrt{2\left\langle\delta \sigma^{2}\right\rangle}<\langle\sigma\rangle$, i.e., if $\eta_{m}>1$, the local violations are extremely rare and the asymmetry function develops a very steep slope. This physical analysis explains why solving Eq. (60) numerically for $\eta_{m} \geqslant 1$

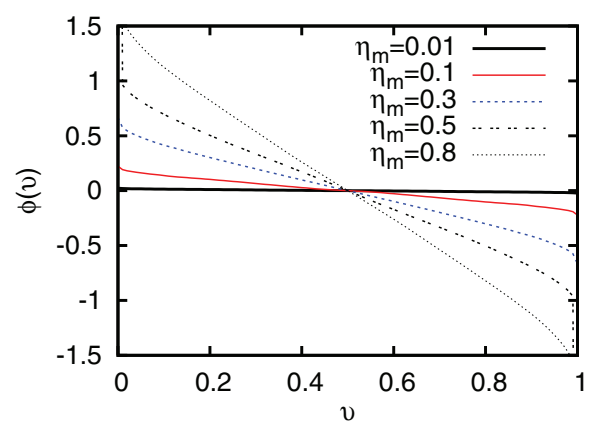

FIG. 4. (Color online) Asymmetry function $\phi(v)$ for $\eta_{m}=0.01$, $0.1,0.3,0.5$, and 0.8 . As $\eta_{m}$ approaches the value $\eta_{m}=1$, the slope of the asymmetry function rapidly becomes very steep. 
and arbitrary values of $s$ is so difficult. In that region, the numerical scheme implemented by us becomes unstable.

\section{DISCUSSION}

We have studied the statistics of the occurrence of entropyconsuming time intervals for single realizations of stochastic processes in phase space. The conditions for the validity of the main results derived here are very general, however, we would like to briefly discuss an important point regarding the definition of the violation fraction itself, which is the central concept in our theory. The average entropy production rate is quite generally well defined, however, its stochastic counterpart may be sometimes ill defined. Note that in certain cases, similar to the velocity of a Brownian walker, the stochastic entropy production rate is well defined only under an integration sign, i.e., in the sense of generalized functions. The stochastic entropy production $S$, on the other hand, is always well defined. However, one can introduce a coarse-grained entropy production rate by considering an arbitrarily small, but still finite, time window $\tau_{w}$. Generalizing the definition given by Eq. (9) to this case, we may write

$$
v\left(\tau, \tau_{w}\right)=\frac{1}{\tau-\tau_{w}} \int_{0}^{\tau-\tau_{w}} \Theta\left(\frac{S(t)-S\left(t+\tau_{w}\right)}{\tau_{w}}\right) d t .
$$

The limit $\tau_{w} \rightarrow 0^{+}$, if it exists, corresponds to the definition given by Eq. (9). It turns out that, using $v\left(\tau, \tau_{w}\right)$ as defined by Eq. (76) instead of our original definition, one can easily prove that all the results we have derived in this paper continue to be valid.

Our analysis is different from the kind of study currently considered in the literature. Instead of focusing on the statistical properties of the final value of the stochastic entropy production at the end of a given protocol, we have considered the whole evolution of the stochastic entropy production rate within the time interval. Even when both approaches are clearly different, they are closely related. Consider, for instance, a system with many degrees of freedom or in an asymptotic steady regime. In both cases the probability density functions of both the violation fraction and the stochastic entropy production $S$ are concentrated around their respective means. In these scenarios, a large-deviation function exists for both quantities. We then have in those cases

$$
\begin{aligned}
\langle S(\tau)\rangle & =\int_{0}^{1}\langle S(\tau) \mid v\rangle \rho(v, \tau) d v \approx\langle S(\tau) \mid\langle v(\tau)\rangle\rangle, \\
\langle v(\tau)\rangle & =\int_{-\infty}^{\infty}\langle v(\tau) \mid S\rangle P(S, \tau) d S \approx\langle v(\tau) \mid\langle S(\tau)\rangle\rangle,
\end{aligned}
$$

where the second relations in Eqs. (77) and (78) follow from the saddle-point evaluation of the corresponding integrals. Thus, there is a one-to-one correspondence between the mean value of the entropy production and the violation fraction, i.e., by controlling one of these quantities it is possible to control the other.

We discuss now a possible application of our results for freeenergy recovery in single-molecule experiments. Note that, for a system initially prepared in a given equilibrium steady state and using the definition of $\Phi$ [Eq. (15)], we can write

$$
\Phi(v, \tau)=\Psi(v, \tau)-\beta \Delta F(\tau),
$$

where

$$
\Psi(v, \tau)=-\ln \left\langle e^{-\beta W(\tau)} \mid v\right\rangle,
$$

$\Delta F$ is the change of the free energy during the protocol, and $\beta$ corresponds to the inverse temperature. Then, for example, from Eq. (3) we can write

$$
\beta \Delta F(\tau)=-\ln \left\langle e^{-\Psi(v, \tau)}\right\rangle .
$$

An even more precise method is to consider Eq. (79). Using the fact that $\Phi(1 / 2, \tau)=0$, we have

$$
\beta \Delta F(\tau)=\Psi(1 / 2, \tau) .
$$

The conceptual problem is that one needs to unmask the behavior of the function $\Phi$ (and correspondingly $\Psi$ ), but the advantage comes from the experimental (or computational) side. Note that $v$ only depends on the instantaneous sign of the entropy production rate, i.e., one does not need its value and it is sufficient to measure the relative orientation of a velocity with respect to a probability current (this determines, quite generally, the sign of $\sigma$ ). Although one still needs to measure $W$ at the end of the interval in order to have an independent measure of $\Psi$ [see Eq. (80)], adding the violation fraction in the analysis could help reduce the error in the estimation of free energies from single-molecule experiments. We believe that the discussion above is interesting enough as to motivate the study of the statistics of the violation fraction in more detail.

Although the analytical treatment of these problems may prove difficult, there is a great deal of accumulated knowledge we can borrow from the study of the zero-crossing properties of generic stochastic processes. This kind of study could create an opportunity for new and fruitful collaborations between different fields of statistical mechanics.

\section{CONCLUSION}

We have studied the statistics of the occurrence of entropyconsuming events for single trajectories of processes in phase space. We were able to obtain a symmetry relation for the duration of these events, which is reminiscent of fluctuation theorems and involves an asymmetry function that has been studied and characterized within this work. We have studied analytically the steady-state limit of this symmetry for a paradigmatic model system, showing that even in the simplest cases it is difficult to determine much analytically. However, we believe, as discussed above, that our study could be of experimental (and computational) relevance, for instance, for the free-energy recovery in single-molecule experiments.

\section{ACKNOWLEDGMENTS}

This work was supported by CNEA, CONICET (Grant No. PIP11220090100051), and ANPCYT (Grant No. PICT20111537). R.G.G. thanks V. Lecomte for valuable comments during the early stages of preparation of the present manuscript, G. Schehr and A. Rosso for providing Refs. [30-32], and Y. Núñez-Fernández for fruitful discussions. 
[1] U. Seifert, Rep. Prog. Phys. 75, 126001 (2012).

[2] D. J. Evans, E. G. D. Cohen, and G. P. Morriss, Phys. Rev. Lett. 71, 2401 (1993).

[3] G. Gallavotti and E. G. D. Cohen, Phys. Rev. Lett. 74, 2694 (1995).

[4] J. Kurchan, J. Phys. A: Math. Gen. 31, 3719 (1998).

[5] J. L. Lebowitz and H. Spohn, J. Stat. Phys. 95, 333 (1999).

[6] C. Jarzynski, Phys. Rev. Lett. 78, 2690 (1997); Phys. Rev. E 56, 5018 (1997).

[7] G. E. Crooks, J. Stat. Phys. 90, 1481 (1998); Phys. Rev. E 61, 2361 (2000).

[8] T. Hatano and S.-i. Sasa, Phys. Rev. Lett. 86, 3463 (2001).

[9] V. Y. Chernyak, M. Chertkov, and C. Jarzynski, J. Stat. Mech. (2006) P08001.

[10] R. García-García, D. Domínguez, V. Lecomte, and A. B. Kolton, Phys. Rev. E 82, 030104(R) (2010).

[11] R. García-García, V. Lecomte, A. B. Kolton, and D. Domínguez, J. Stat. Mech. (2012) P02009.

[12] T. Speck and U. Seifert, J. Stat. Mech. (2007) L09002.

[13] T. Ohkuma and T. Ohta, J. Stat. Mech. (2007) P10010.

[14] T. Mai and A. Dhar, Phys. Rev. E 75, 061101 (2007).

[15] C. Aron, G. Biroli, and L. F. Cugliandolo, J. Stat. Mech. (2010) P11018.

[16] F. Zamponi, F. Bonetto, L. F. Cugliandolo, and J. Kurchan, J. Stat. Mech. (2005) P09013.

[17] A. V. Chechkin and R. Klages, J. Stat. Mech. (2009) L03002.

[18] R. García-García, Phys. Rev. E 86, 031117 (2012).

[19] G. M. Wang, E. M. Sevick, E. Mittag, D. J. Searles, and D. J. Evans, Phys. Rev. Lett. 89, 050601 (2002).
[20] E. H. Trepagnier, C. Jarzynski, F. Ritort, G. E. Crooks, C. Bustamante, and J. Liphardt, Proc. Natl. Acad. Sci. U.S.A. 101, 15038 (2004).

[21] C. Bustamante, J. Liphardt, and F. Ritort, Phys. Today 58(7), 43 (2005).

[22] D. Carberry, M. Baker, G. Wang, E. Sevick, and D. J. Evans, J. Opt. A 9, S204 (2007).

[23] F. Ritort, Adv. Chem. Phys. 137, 31 (2008).

[24] A. N. Gupta, A. Vincent, K. Neupane, H. Yu, F. Wang, and M. T. Woodside, Nat. Phys. 7, 631 (2011).

[25] R. García-García and D. Domínguez, Phys. Rev. E 89, 022116 (2014).

[26] M. Esposito and C. Van den Broeck, Phys. Rev. Lett. 104, 090601 (2010).

[27] M. Esposito and C. Van den Broeck, Phys. Rev. E 82, 011143 (2010); C. Van den Broeck and M. Esposito, ibid. 82, 011144 (2010).

[28] I. Dornic and C. Godreche, J. Phys. A: Math. Gen. 31, 5413 (1998).

[29] S. R. Williams, D. J. Evans, and D. J. Searles, J. Stat. Phys. 145, 831 (2011).

[30] M. Densing, Phys. A 391, 5818 (2012).

[31] S. N. Majumdar and A. J. Bray, Phys. Rev. E 65, 051112 (2002).

[32] A. J. Bray, S. N. Majumdar, and G. Schehr, Adv. Phys. 62, 225 (2013).

[33] F. M. Fernández, Eur. J. Phys. 31, 961 (2010).

[34] W. Mei and Y. Lee, J. Phys. A: Math. Gen. 16, 1623 (1983).

[35] M. Abramowitz and I. A. Stegun, Handbook of Mathematical Functions (Dover, New York, 1970). 